\title{
Use of White Light Interference Methods in the Opaque Mediums Diagnostic
}

\author{
J. KasprzaK*, A. Borkowska And M. KeCIK \\ Department and Ophthalmology Clinic, I Medicine Faculty, Medical University of Warsaw \\ W. Lindleya 4, 02-005 Warsaw, Poland
}

\begin{abstract}
The reflection, absorption and scattering influence on turbid structure has been discussed. The influence of transverse scanning method on geometrical deformations as well as issues of thickness absolute measurements and refractive index evaluation methods has been analyzed. A number of phenomena which may be the artefact source and the cause of tomogram incorrect interpretation have been pointed out.
\end{abstract}

PACS: 47.63.Cb, 87.19.U-, 07.05.Tp

\section{Introduction}

Optical coherence tomography (OCT) method permits precise, non-invasive and non-contact imaging of tissue structures. The basic system used in the white light interferometry is the system being the Michelson interferometer modification [1-3]. The application of the radiation characterized by low coherence in coherent tomography permits the measurement of medium optical properties from the layers whose thickness is close to the light source coherence path value.

The OCT technique has been used in the eye disease diagnostic since 1996. The first devices utilizing this technique were designed for performing retina tomograms. The clinical material acquired fully confirmed the enormous usefulness of this technique in imagining of an eyeball rear section. Currently, the OCT tomogram analysis results in many retina affections may be treated as decided [4-6].

Specific features of the OCT technique may be used for the need of non-standard clinical tests. In ophthalmology, the natural direction of clinical application extension was the imaging tests of an eyeball front section. Based on the optical fiber interferometers, the OCT method can be identified with various types of catheters, endoscopes and devices for laparoscopy, which permits wide application of this diagnostic technique in many branches of medicine [6-10].

The main issue of medical techniques of imagining is the detection of micro and macro inhomogeneities of organism internal structures. Tissues are multicomponent structures characterized by different arrangement ratio and peculiar spectral characteristics. The most important factors influencing light propagation in tissues are

\footnotetext{
* corresponding author; e-mail: kasprzak@amwaw.edu.pl
}

reflection, scattering and absorption. The object geometry and the influence of surrounding tissues are also very important [11].

\section{Assessment of the refraction, absorption and scattering coefficients influence on tomogram quality}

Collimated light beam transmission through the tissue of $d$ thickness is described by Lambert-Beer's law

$$
I(d)=\left(1-R_{\mathrm{F}}\right) I_{0} \exp \left(\mu_{\mathrm{e}} d\right),
$$

where $I(d)$ - light intensity transmitted through a tissue layer of $d$ thickness, $I_{0}$ - light intensity incident on boundary surface, $\mu_{\mathrm{e}}=\mu_{\mathrm{a}}+\mu_{\mathrm{e}}$ - extinction coefficient being the sum of an absorption coefficient $\mu_{\mathrm{a}}$ and a scattering coefficient $\mu_{\mathrm{r}} . R_{\mathrm{F}}-$ Fresnel's refractive index.

The Fresnel refractive index $R_{\mathrm{F}}$ on the boundary of two mediums depends on the subtraction of their refractive indexes $n_{1}$ and $n_{2}$ in the following way:

$$
R_{\mathrm{F}}=\left[\left(n_{1}-n_{2}\right) /\left(n_{1}+n_{2}\right)\right]^{2} .
$$

In case of the reflection from boundary surfaces within an eyeball, the refractive index differences of internal structures are not big. As a result, the refractive index values do not exceed $3 \%$. In the spectral range of $0.6-1.5 \mu \mathrm{m}$, the backscattering is the factor that increases the intensity of the radiation reflected. The value of the extinction coefficient (for both reflected and scattered light) in this spectral range may exceed $70 \%$.

In OCT detection, when the backscattered radiation intensity value is high, it is possible to observe the undesirable phenomena in the form of apparent images located in the space both in front of and behind boundary surface. A space distribution of these images is dependent on the reflective surface distance. This phenomenon 
was most frequently observed during the research on flat-parallel, transparent plates located in the air. In case of thin, multilayer samples the superposition of apparent and real images of extreme surfaces was observed (Fig. 1b).

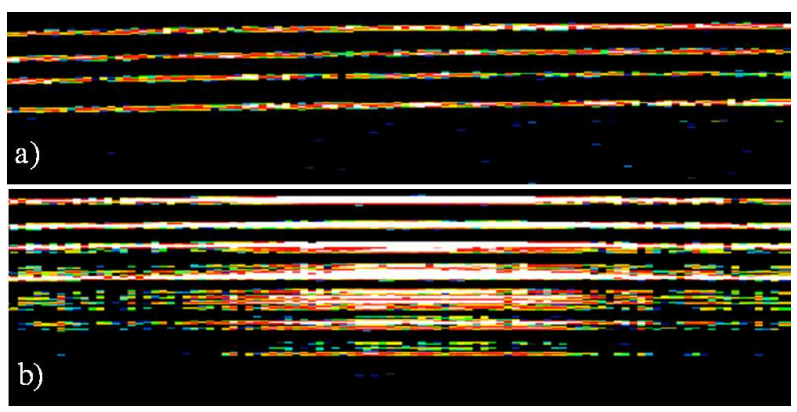

Fig. 1. The tomogram obtained during the perpendicular falling of the diagnostic light beam. Tomogram without reflections (a). Artefacts caused by multiple reflections for thin, multilayer structures (b).

In the intravital research, the artefacts accompanying the increased reflection are most often observed when examining cornea. Soft distortions of homogeneity of the optical signal in the area of the vitreous body are sporadically observed in retina tomograms done in case of eyes characterized by strong reflections from the eyeground (Fig. 2).
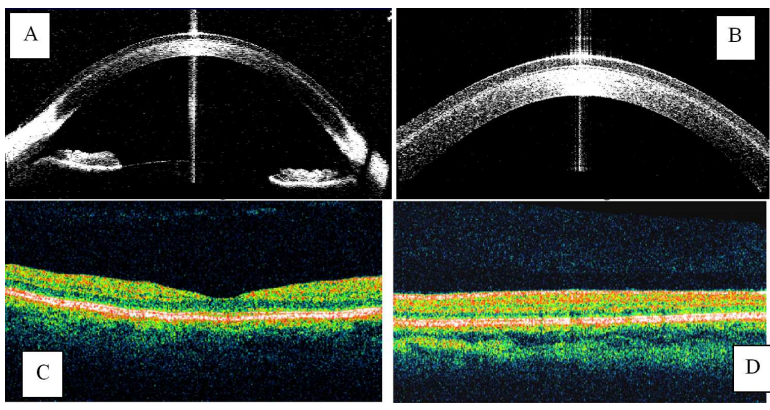

Fig. 2. Artefacts caused by reflection from the boundary surfaces in the eye. (A), (B) Artefacts caused by reflection in the top part of the cornea, observed in tomograms obtained by means of VISANTE device. (C), (D) Falsely positive subtle changes in the area of the vitreous body in retina tomograms carried out by means of VISANTE device in healthy eyes with strong reflection from the eyeground.

Unfavorable effects connected with the reflection and backscattering reveal strong dependence on the incidence angle. Measurement light beam shift increasing the angle in the range of a few degrees can totally reduce this phenomenon. However, in case of turbid surfaces without scattering centers the use of reflection is in principle the only method permitting the unambiguous identification of surfaces examined. However, the contrast imaging may be obtained at perpendicular setting of the light beam to the sample surface. This phenomenon is very crucial to the thickness measurement of the cornea. This issue is more precisely illustrated in Fig. 3. The comparative measurements were done by means of STRATUS device - for the transparent test glass plate and strongly scattered frosted sample.

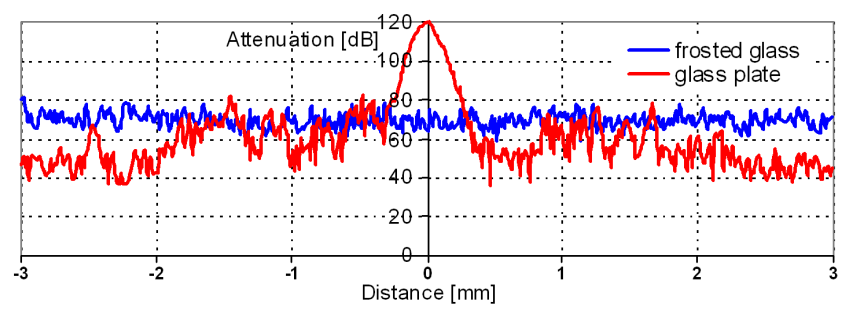

Fig. 3. Comparison of maximum values of the optical signal amplitude for the boundary surfaces of the structures in the tomograms done for the frosted glass and the transparent glass surface.

For both surfaces linear scans of $6 \mathrm{~mm}$ length were done. Maximum signal values along the scan are presented in the graph. The center of the graph corresponds to the axis of the sector scanning. In accordance with the expectation, in case of scattering surface the light intensity recorded, in fact, did not depend on the distance from the axis of the scanning sector. The signal values revealed strong fluctuations. In case of the reflecting surface (glass plate) the character of the optical signal was different. For extreme points the optical signal value is definitely lower whereas for the points located centrally there a distinct decrease in the signal fluctuation occurs.

The OCT technique is applied to the imaging of the tissue structure with intermediate values of the scattering coefficient. Too low scattering values make the structure reconstruction difficult, however, high values definitely limit the penetration depth of the diagnostic beam (Fig. 4).
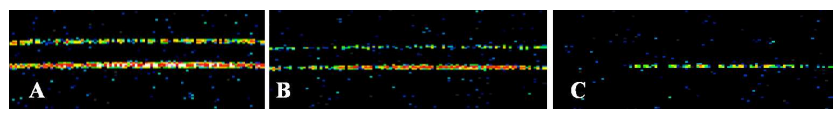

Fig. 4. Tomograms of the same sample during the increasing concentration of the scattering centers of the surrounding medium: water $(\mathrm{A})$, dilute milk 1:100 (B), dilute milk - 1:30 (C).

In mediums revealing strong scattering a tomogram structure is a result of multiple reflections from scattering centers of the external layers characterized by the thickness of a few dozen $\mu \mathrm{m}$. These layers obscure the structures located deeper. For strong turbidities on the beam path, the scattering issue may be a difficult problem to solve (Fig. 5).

In ophthalmologic applications cornea scars, cataract, changes in a vitreous body may be factors making the beam penetration difficult and in an extreme case can make the OCT examination impossible. 


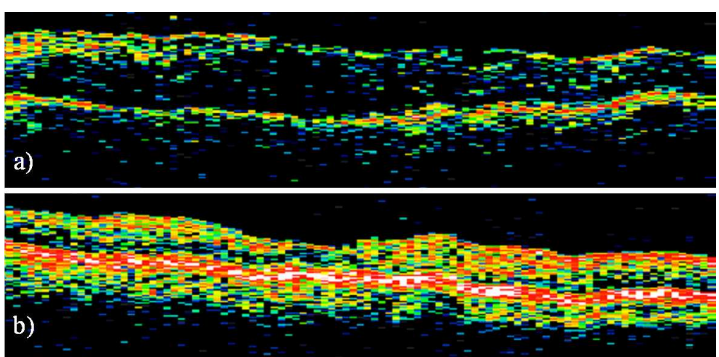

Fig. 5. A tomogram of the retina of a patient with turbid optical mediums (a) and retina tomogram in the concomitant eye.

Absorption is also a very important factor - when attenuating the beam, it decreases the image contrast. The absorbing structures located on the beam path cause blackouts blanking off structures located deeper. As a result of that, an optical signal amplitude and an optical signal contrast decrease. In clinical conditions structures characterized by high extinction may constitute a crucial problem - limiting the possibility of tomogram interpretation exclusively for external layers (Fig. 6).

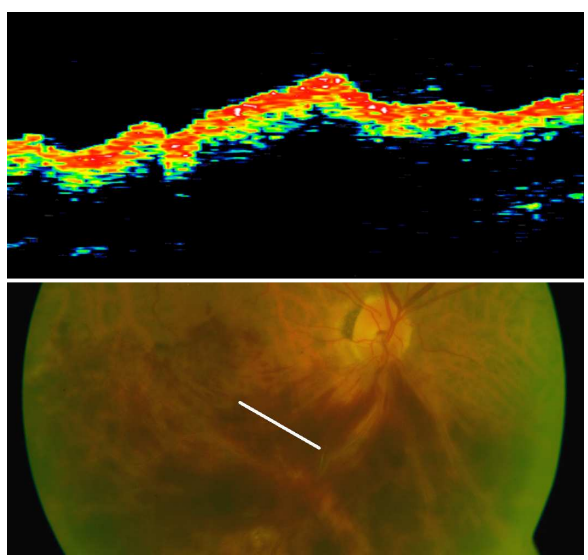

Fig. 6. A tomogram of the retina with the closing of vessels caused by a clot. On the eyeground image the tomogram location (white line) was marked.

The attenuation of the optical signal may be also observed in a situation when the beam is angled to the boundary surfaces. For tomograms of the rear pole of the eyeball, skew beam setting to the surfaces of the structures examined may occur within large vessels, on the verge of the optic nerve disc as well as in pathologies occurring in the form of a sudden rise of the retina.

\section{Medium refractive index influence on the thickness measurement}

Algorithms used in ophthalmologic OCT systems are adapted for retina thickness measurement. Wishing to use these devices for the structure measurements other than retina it is necessary to determine the refractive index $\left(n_{\mathrm{a}}\right)$ value given by the producer of a device. Another important issue is to know the refractive index of tissues examined. In algorithms available, the $n_{\mathrm{a}}$ index value (applied to conversion of an optical length into a geometrical length) is not given in engineering specification. The value of this coefficient can be estimated experimentally applying specific features of the method.

In commercial solutions the structure tomogram is presented in a measuring window. Here, the space relations in axial direction result from the optical length both surrounding the medium and the sample. As a result of light delay in mediums (on the beam path) the shifting of sample image in axial direction is observed. However, the refractive index change within the sample affects the location of the next boundary surfaces of the internal structures changing the reading of geometrical relations from a tomogram.

The refractive index values applied in accessible devices can be defined by comparing the real distance $d$ of the boundary surfaces of the plates characterized by known refractive index $n$ with the length measurement $l_{\mathrm{a}}$ made with the use of the apparatus. Comparing the relations describing optical path difference $\Delta z$ for the sample examined (taking refractive index $n$ and real thickness $d$ into consideration) given as

$$
\Delta z=2 n d
$$

with the relation obtained on the basis of the apparatus measurements (for the case when the beam double passes through the sample):

$$
\Delta z=2 n_{\mathrm{a}} l a
$$

the following relation is obtained:

$$
n_{\mathrm{a}}=\frac{n d}{l a} .
$$

As results from (5), the refractive index $n_{\mathrm{a}}$ value can be determined by comparing the shift of the tomogram edge measured in measuring window with the shift of the test plate in a reference arm (the refractive index value for air $n=1$ was taken). Using this method, the $n_{\mathrm{a}}$ coefficient values for both OCT1 system $\left(n_{\mathrm{a}}\right.$ OCT1 $\left.=1.407\right)$ and STRATUS system $\left(n_{\text {a STRATUS }}=1.378\right)$ were determined. Literature values [12] of the refractive index for human retina measured in vitro for the selected wavelength from the range of 450-600 nm equaled from 1.34 to 1.38. Knowing the refractive index defined by the producer, it is possible to measure the refractive index of samples characterized by the given thickness.

\section{Tomogram distortion caused by geometry of the scanning sector}

For the sake of the specific eyeball structure in ophthalmologic OCT systems designed for retina examination the sector scanning method is applied. The light rays passing the frontal nodal point $n_{\mathrm{p}}$ pass through the optical system of the eye and come out of the rear nodal point $n_{\mathrm{t}}$ without the direction change - which allows 
calculating the real length of the tomogram at the eyeground (Fig. 7). During measurements the laser beam scans a certain sector at an angle depending on the scan length. In case of long tomograms made for flat samples, the characteristic structure deformation resulting from geometrical relations within the scanning sector is observed (Fig. 8).

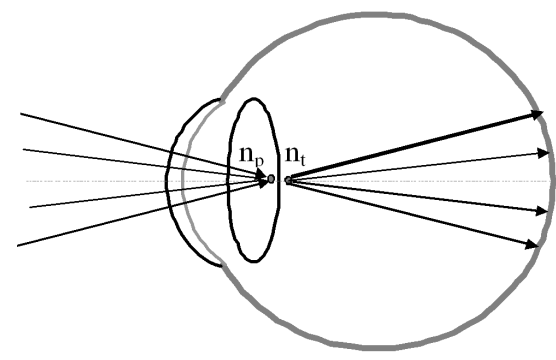

Fig. 7. A diagram of optimum light ray path in case of the sectorial scanning of the retina.

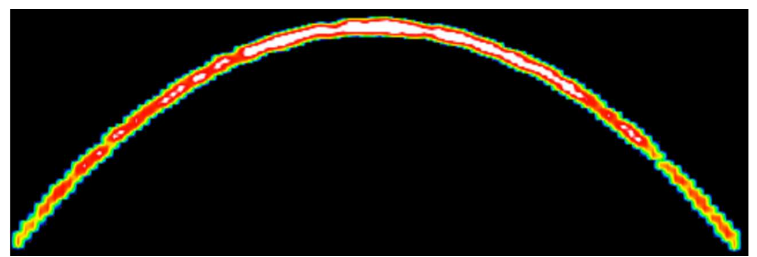

Fig. 8. A deformation of the tomogram plane at a big angle of the scanning sector (OCT1).

The tomogram deformation observed is a result of the optical path lengthening accompanying the change of the angle of the measuring beam during the scanning of the particular scanning sector (Fig. 9).

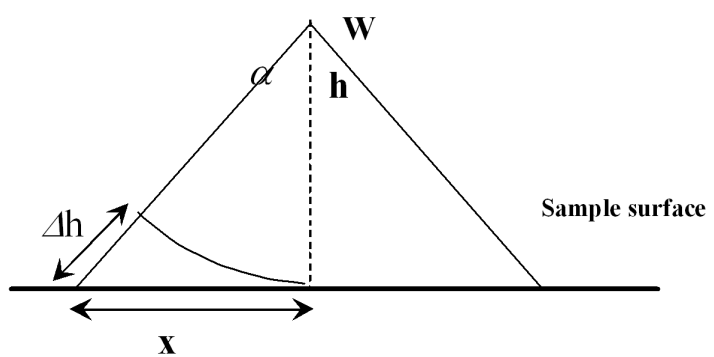

Fig. 9. An optical path lengthening - the cause of the tomogram deformation.

The shifting values of individual elements of the plane can be described by means of simple geometrical relation

$$
\Delta h=\left(x^{2}+h^{2}\right)^{1 / 2}-h,
$$

where $\Delta h-$ the value of geometrical deformation, $x$ the distance of the point from scanning sector axis, $h-$ the distance of the surface from the top of the scanning sector $W$
Performing measurements of the deformation values $\Delta h$ for selected distances $x$, the distance $h$ corresponding to the scanning sector height can be estimated. The $x$ value normalization requires the consideration of transverse magnification.

\section{Summary}

Devices utilizing the OCT technique permitting precise, non-invasive and non-contact imaging of tissue structures make up a very attractive diagnostic tool. In case of attempts at expanding of the application range of this technique, the users have to be conscious of many dangers.

Doing research with the use of physical models allowing modifying selected optical features constitutes an excellent way of gaining clinical experience by the users of the OCT technique. Gaining experience makes the tomogram interpretation easier and also allows extending the application range of the method. Techniques permitting automatic optimization of the optical signal (proposed in the newest constructions) are very helpful. After the tomogram recording, its quality is automatically evaluated. Boundaries fitting uncertainty in algorithms allowing geometrical analyzing of the structures is also evaluated. In spite of that, fitting errors, particularly in case of tissues characterized by atypical structural changes are observed (Fig. 10).

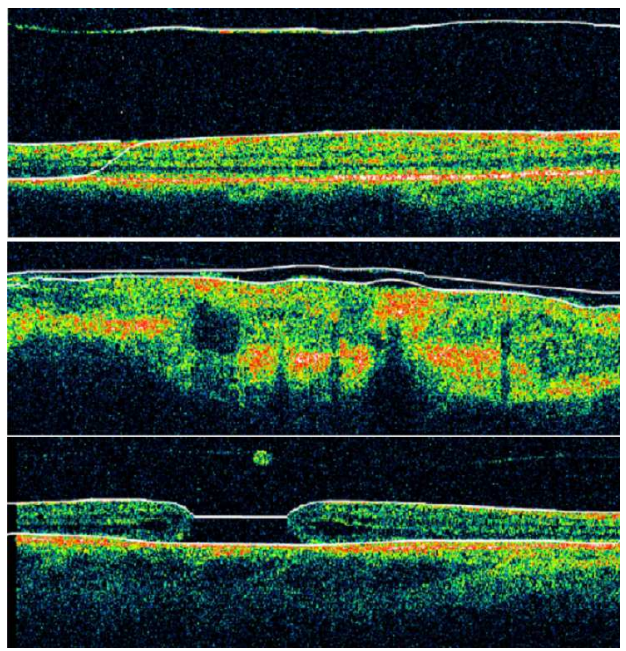

Fig. 10. An example of incorrect matching of the structures examined in the retina tomograms. Automatic tomogram quality evaluation was close to maximum values.

The examples presented show that procedures occurring in commercial devices continuously reveal a number of shortcomings. Doctors' expectations are still ahead of the available possibilities in a commercial offer. For instance, in case of tomographic examination of the retina, doctors wait for the possibilities of an analysis taking multilayer internal retina structure into account and es- 
pecially procedures allowing evaluating the evolution of spot holes.

\section{References}

[1] D. Huang, E.A. Swanson, C.P. Lin, J.S. Schuman, W.G. Stinson, W. Chang, M.R. Hee, T. Flotte, C.A. Puliafito, J.G. Fujimoto, Science 254, 1178 (1991).

[2] M.R. Hee, J.A. Izatt, E.A. Swanson, D. Huang, J.S. Schuman, C.P. Lin, C.A. Puliafito, J.G. Fujimoto, IEEE Eng. Med. Biol. 1/2, 67 (1995).

[3] Y. Pan, E. Lankenau, J. Welzel, R. Birngruber, R. Engelhardt, IEEE J. Sel. Topics Quant. Electr. 2, 1029 (1996).

[4] D.S. Chauban, J. Marshal, Investigative Ophtalmology Vis. Sci. 40, 2332 (1999).

[5] C.A. Toth, D.G. Narayan. A.A. Boppart, M.R. Hee, J.G. Fujimoto, R. Birnguber, C.P. Cain, Ch. DicCarlo, W. Roach, Archiv. Ophthalmol. 115, 1425 (1997).
[6] S. Muscat, S. Parks, E. Kemp, D. Keating, Investigative Ophtalmology Vis. Sci. 43, 490 (2002).

[7] E. Grube, U. Gerckens, L. Buellesfeld, P.J. Fitzgerald, Circulation 106, 2409 (2002).

[8] G.J. Tearney, M.E. Brezinski, B. Bauma, S. Boppart, C. Pitris, J. Southern, J.G. Fujimoto, Science $\mathbf{2 7 6}$, 2037 (1997).

[9] J.A. Izatt, M. Kulkarni, H.W. Wang, K. Kobayashi, M. Sivak, IEEE J. Quant. Electron. 2, 1017 (1996).

[10] M. Gawlikowski, T. Pustelny, B. Przywara-Chowaniec, J. Nowak-Gawlikowska, Acta Phys. Pol. A 118, 1124 (2010).

[11] Tissue Optics: Applications in Medical Diagnostics and Therapy, Ed. V.V. Tuchin, MS102, SPIE Press, Bellingham 1994.

[12] D. Sardar, R. Yow, A. Tsin, R. Sardar, J. Biomed. Opt. 10, 051501 (2005). 